\title{
For unitary "theory" of Chemometrics
}

$\mathrm{N}$ ow, at the beginning of this new millenium, "Chemometrics" is widely known among Scientists. But when a Chemist or a so-called Chemometrician is required to give a short definition of this new specialty, the answer is far from to be spontaneous! From a round table enquiry, it was shown that most answers are biased according to the two main application fields of involved people: (i) Experimental Design (ED) or (ii) Data Processing (DP). Only seldom definitions focussed on a unitary view of chemometrics, such as "The science of processing the chemical information"! Chemometrics at the early beginning, about three decades ago, was supported mainly by development in DP, more particularly with factor analysis. Whereas diffusion of Chemometrics inside the scientific community, in the last decade, was largely supported by industrial applications based on ED for process optimization.

In fact the fuzzy frontier between ED and DP lies on the role devoted to the chemical compound itself. In ED the compound is no more than an object, an element of a puzzle, e.g: in formulation for new inorganic materials or in process optimization. Whereas, in DP, the compound is the object of precise studies, e.g. or as a complex 3D structure when analyzing subtle ligand/receptor interactions in Bioinformatics.

Presently, in this early stage of this millenium, we really feel that the gap between ED and DP would disappear. In fact we are facing a paradoxical situation! With more and more in depth studies in ED and EP procedure refinements, the complexity in both areas is increasing and simultaneously unitary approach to Chemometrics is developing! The gap between ED and DP is essentially sensitive when the stress is put on the involved application fields. But when the attention is focussing on the methodology, the unitary view of chemometrics increases drastically. The unity of concepts is supported by the following points: (i) model robustness (ii) model visualization to suggest the best optimization, (iii) more powerful mathematical procedures.

It is precisely the interest of the symposium "Chimiométrie 99", which was held in Paris, on December 2-3, 1999, to encourage scientific and human communication around different facets of a same discipline and promote a unitary view for Chemometrics. This thematic issue of Analusis - European Journal of Analytical Chemistry, entitled "Chemometrics 2000", with some selected contributions issued from the above symposium, gives simultaneously an overview of the large diversity and strong unity of Chemometrics, insofar the stress is put on concepts and procedures rather than on the application fields. So, all the authors of this special issue of Analusis, the contributors of the above symposium and more particularly the Société de Chimie Industrielle, the Société Française de Chimie, the Publisher EDP Sciences/Wiley-VCH and the Editor in Chief of the Journal, have to be acknowledged for their valuable contribution in encouraging this "Unitary 'Theory' of Chemometrics"! 


\section{Chemometrics 2000}

561 For unitary "theory" of Chemometrics J.R. Chrétien

563 Boolean experimental designs J. Goupy

571 Central composite design and optimization by multiresponse analysis of octacalcium phosphate synthesis J.-C. Heughebaert, A. Lebugle, F. Vu and R. Phan-Tan-Luu

575 Desirability approach for optimisation of electrothermal atomic absorption spectrometry factors in iron determinations

S. Salomon, P. Giamarchi and A. Le Bihan

587 Experimental design optimization of chromatographic separation for polycyclic aromatic hydrocarbons in vegetable oils A.-M. Dorthe, J.-L. Ramberti and A. Thienpont

592 Development of an HPLC method for the determination of phenolic by-products: optimisation of the separation by means of the experimental designs methodology B. Motamed, J.-L. Böhm, D. Hennequin, H. Texier, R. Mosrati and D. Barillier

600 Modeling properties of water-based paints dried by microwaves from the design and analysis of a mixture-process experiment S. Barra, F. Boulanger, A. Trapani and D. Cabrol-Bass

610 Characterisation of the staphylococcal enterotoxin research method in a dairy product L. Macaluso and C. Lapeyre

616 "Chemometrics" applied to the optimization of the preparation of hydrotopes for detergents starting from BTX fraction of natural gas

A. Kamoun and M. Châabouni

625 Prediction of odours of aliphatic alcohols and carbonylated compounds using fuzzy partition and self organising maps (SOM)

K. Audouze, F. Ros, M. Pintore and J.R. Chrétien

633 Quantitative gas detection with semiconductor micro-sensors and chemometrics P. Breuil, N. Perdreau and C. Pijolat

637 3D quantitative structure activity relationships with CoRSA. Comparative receptor surface analysis. Application to calcium channel agonists

$O$. Ivanciuc, T. Ivanciuc and D. Cabrol-Bass

Cover Design: Bacteriorhodopsin homologous to olfactive receptors and map organisation of an olfaction data base after K. Audouze, F. Ros, M. Pintore, J.R. Chrétien p. 625 of the present issue. 